\title{
ANÁLISE DA APLICAÇÃO DO APRENDIZADO BASEADO EM JOGOS NO CONTEXTO REMOTO: O CASO DA DISCPLINA DE PROJETO E CONSTRUÇÃO DA INFRESTRUTURA VIÁRIA
}

DOI: 10.37702/2175-957X.COBENGE.2021.3667

BRUNO CAVALCANTE MOTA - bruno86925@gmail.com

UNIVERSIDADE FEDERAL DO CEARÁ

Rua Padre Alzir Sampaio 371

60355-260 - Fortaleza - CE

SABRINA RODRIGUES GONCALVES - sabrinarg57@gmail.com

Universidade Federal do Ceará

Rafael Dantas 2236

60830-585 - Fortaleza - CE

FRANCISCO HEBER LACERDA DE OLIVEIRA - heber@det.ufc.br UNIVERSIDADE FEDERAL DO CEARÁ

Centro de Tecnologia Bloco 703

60440-900 - Fortaleza - CE

JAIR CARVALHO FREIRE - jaircarvalhofreire123@gmail.com

UNIVERSIDADE FEDERAL DO CEARÁ

Avenida XXVI Res. Pacatuba II B 29 Ap 104

61800-000 - Pacatuba - CE

Evanilson de Lima Siqueira - evanilsondelimasiqueira@gmail.com

Universidade Federal do Ceará

Rua Dom Xisto Albano 3854

60730-225 - Fortaleza - CE

Resumo: No contexto ensino-aprendizagem de engenharias, tem surgido cada vez mais debates que questionam os modelos tradicionais e meramente expositivos de ensino. Debates acerca do protagonismo do estudante nas aulas e a motivação dos discentes se tornam ainda mais relevantes no contexto provocado pela pandemia da COVID-19. Com a suspensão das aulas presenciais, professores e alunos foram obrigados a migrar para plataformas online, dando prosseguimento 
as atividades didáticas através do ensino remoto. No centro desses debates, tem-se a ascensão de diversas metodologias de ensino que buscam envolver os estudantes, colocando-os como protagonistas do aprendizado. Nessa linha, destaca-se neste trabalho a Aprendizagem Baseada em Jogos, metodologia referente ao uso do poder de motivação e entretenimento de jogos voltada para o ensino. Dessa forma, o objetivo deste artigo é compartilhar uma aplicação da estratégia pedagógica de aprendizagem baseada em jogos e avaliar sua contribuição em uma turma da disciplina de Projeto e Construção da Infraestrutura Viária (PCIV) ofertada no modelo totalmente remoto em 2020. Para a aplicação dos jogos foi utilizada a ferramenta kahoot de aplicação de testes rápidos de múltipla escolha acerca do conteúdo visto na disciplina. Os resultados reforçam a percepção positiva dos estudantes acerca da metodologia Aprendizagem Baseada em Jogos uma vez que os discentes consideraram os jogos divertidos e que as atividades contribuíram para consolidar os assuntos vistos nas aulas.

Palavras-chave: Ensino Remoto. Aprendizagem Baseada em Jogos. Engenharia Civil 


\section{ANÁLISE DA APLICAÇÃO DO APRENDIZADO BASEADO EM JOGOS NO CONTEXTO REMOTO: O CASO DA DISCPLINA DE PROJETO E CONSTRUÇÃO DA INFRESTRUTURA VIÁRIA}

\section{INTRODUÇÃO}

No contexto ensino-aprendizagem de engenharias, tem surgido cada vez mais debates que questionam os modelos tradicionais e meramente expositivos de ensino. Para Bardini e Spalding (2017), a nova geração de estudantes é usualmente confrontada com um ensino clássico e tradicional, o que pode não apenas desfavorecer a relação professoraluno, mas comprometer o processo entre ensinar e aprender. Para Damasceno (2019 apud MARQUES E GITAHY, 2017) essas aulas expositivas, com métodos de ensino tradicionais, com o docente no centro do conhecimento, faziam mais sentido quando o acesso à informação era difícil.

Buscando reverter esse cenário, tem-se a ascensão de diversas metodologias de ensino que buscam envolver os estudantes, colocando-os como protagonistas do aprendizado. Guimarães (2020) defende que para que aconteça o ensino e a aprendizagem é preciso que discentes sejam protagonistas e construam suas próprias respostas a partir de uma aprendizagem significativa.

Debates acerca do protagonismo do estudante nas aulas e a motivação dos discentes se tornam ainda mais relevantes no contexto provocado pela pandemia da COVID-19. Com a suspensão das aulas presenciais, professores e alunos foram obrigados a migrar para plataformas online, dando prosseguimento as atividades didáticas através do ensino remoto. No entanto, em alguns casos, essa migração foi abrupta, despreparada e segue a premissa de que ensinar consiste apenas em transmitir "conteúdo" (GUSSO et al,2020). Para os autores (GUSSO et al, 2020), uma das consequências é a baixa motivação dos estudantes, que pode até aumentar a evasão nos cursos.

Nessa linha, destaca-se neste trabalho a Aprendizagem Baseada em Jogos (GameBased Learning) definida por Prensky (2001) como a estratégia que se refere ao uso do poder de entretenimento dos jogos digitais para fins educacionais. A Aprendizagem Baseada em Jogos é uma metodologia pedagógica que se foca na concepção, desenvolvimento, uso e aplicação de jogos na educação e na formação dos discentes (CARVALHO, 2015).

Para Contreras et al. (2013) o uso de jogos em aulas são estratégias interessantes para o desenvolvimento de competências dos alunos considerando não só a motivação que eles podem gerar, mas também o tempo de diversão que proporcionam. Paiva e Tori (2017) citam como vantagens da aprendizagem baseada em jogos: i) efeito motivador ii) facilitação do aprendizado iii) desenvolvimento de habilidades cognitivas iv) aprendizagem por descoberta e novas identidades v) socialização.

Dessa forma, o objetivo deste artigo é compartilhar uma aplicação da estratégia pedagógica de aprendizagem baseada em jogos e avaliar sua contribuição em uma turma da disciplina de Projeto e Construção da Infraestrutura Viária (PCIV) ofertada no modelo totalmente remoto em 2020.

\section{2 METODOLOGIA}

\subsection{A ferramenta Kahoot}


Para o desenvolvimento da estratégia ABJ no decorrer da disciplina foi utilizada a ferramenta Kahoot, uma plataforma de aprendizado baseada em jogos. A plataforma é online e gratuita sendo possível, dentre outras estratégias, a aplicação de testes no formato de perguntas com múltiplas escolhas que podem ser acessados por meio de um navegador da Web ou do próprio aplicativo Kahoot.

A ferramenta conta também com versões pagas para quem deseja obter outras funcionalidades. Ao preparar os testes pode-se configurar o tempo de duração, se o teste será síncrono ou assíncrono além de ser possível acessar bancos de questões de outros usuários da plataforma.

Nas aplicações síncronas, os alunos visualizavam as perguntas ao vivo na tela do monitor que aplicava o jogo e apareciam para eles os itens de múltipla escolha. No formato assíncrono, os alunos tinham acesso as perguntas e em seus dispositivos e respondiam independentemente do monitor, além de poderem escolher um horário para realizar os testes, pois ele ficava disponível por algumas horas.

Figura 1- Exemplo de aplicação do Kahoot.

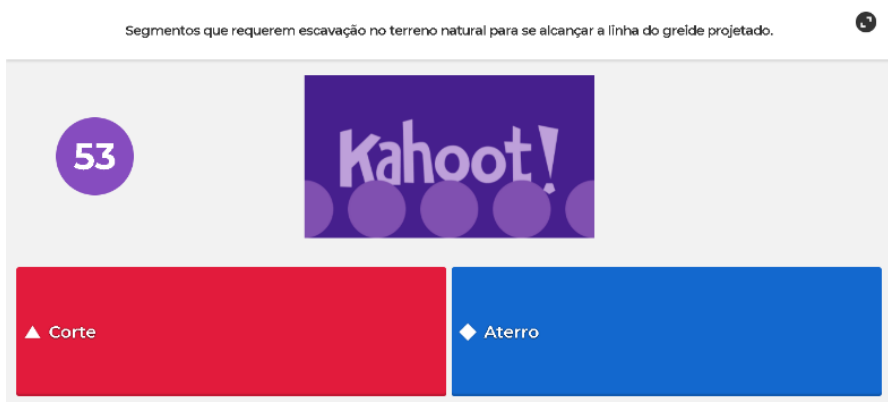

Fonte: Os autores (2021)

\subsection{Aprendizagem baseada em jogos em PCIV}

Projeto e Construção da Infraestrutura Viária (PCIV) é uma disciplina do 6ํㅗ semestre do curso de Engenharia Civil da Universidade Federal do Ceará (UFC), ofertada anualmente. A disciplina percorre todo o processo de projeto de infraestrutura de rodovias, desde a concepção do projeto geométrico ao orçamento e planejamento do projeto de terraplenagem. No semestre 2020.2, PCIV foi ofertada de forma totalmente remota devido à suspensão das aulas presenciais e uma das estratégias utilizadas para engajar os estudantes foi a aplicação da aprendizagem baseada em jogos através do Kahoot.

A disciplina é dividida em 03 etapas (i) projeto geométrico; (ii) projeto de terraplenagem e (iii) custos de serviço de terraplenagem e ao final de cada etapa foram aplicados os jogos que valiam bônus para a nota final. Ao final das duas primeiras aplicações o feedback dos alunos foi captado e ao final da disciplina os discentes respondiam um formulário eletrônico com o intuito de captar a percepção destes acerca dos jogos no aprendizado. O esquema de delineamento desta pesquisa pode ser observado na figura 2 abaixo: 
Figura 2 - Metodologia da pesquisa.

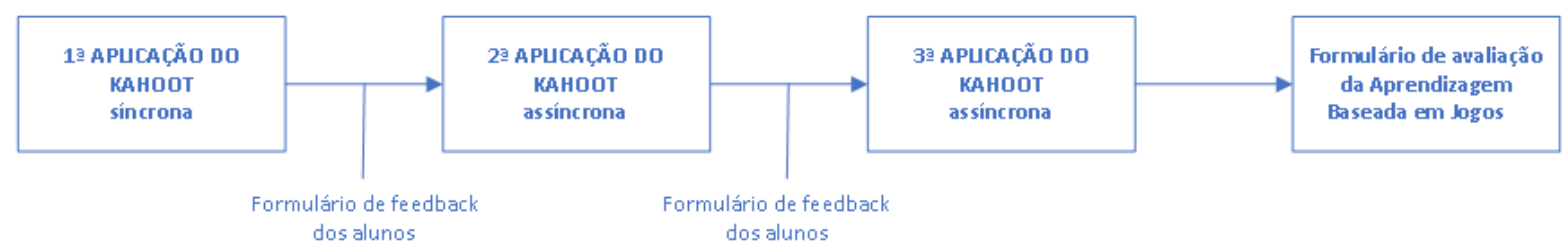

Fonte: Os autores (2021)

\section{RESULTADOS E DISCUSSÃO}

Quando comparada as aplicações do jogo síncrona com assíncrona, os alunos relataram na primeira estratégia que a conexão da internet era um empecilho para a atividade, apesar de a estratégia ser mais dinâmica, por provocar delays entre as informações. No formato assíncrono, esse problema foi pouco relatado.

No formulário de avaliação da Aprendizagem Baseada em Jogos, a primeira questão referia-se à percepção dos estudantes acerca da diversão dos testes no Kahoot. 80\% dos estudantes concordam totalmente que os testes foram divertidos. Essas afirmações também foram captadas através dos feedbacks dos estudantes.

Figura 3 - Diversão na aplicação dos testes Kahoot

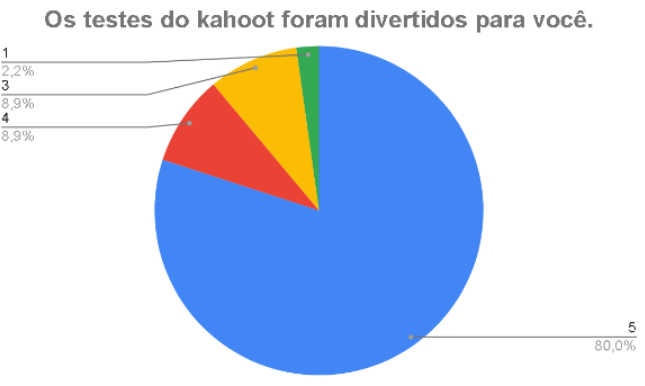

Fonte: Os autores (2021)

Sobre a premiação, os alunos recebiam bônus na nota final de cada etapa com base em seu desempenho na atividade, assim $76 \%$ dos alunos concordam totalmente que isto os incentivou a participar mais das atividades e nenhum aluno discordou da afirmação. Apresentar uma bonificação torna a atividade mais atrativa para os estudantes. 
Figura 4 - Premiação ao final do teste

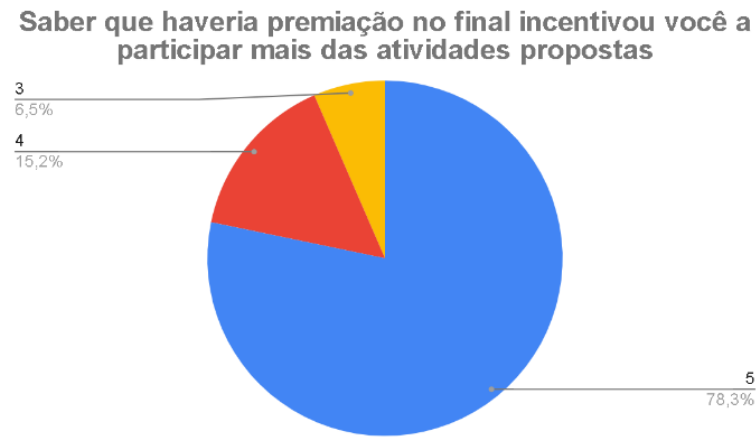

Fonte: Os autores (2021)

Em seguida, os alunos foram questionados se os jogos contribuíram para a aprendizagem e $78 \%$ concordam totalmente que houve contribuição na construção de conhecimento, reforçando uma das premissas da aprendizagem baseada em jogos.

Figura 5 - Contribuição dos jogos aplicados no aprendizado.

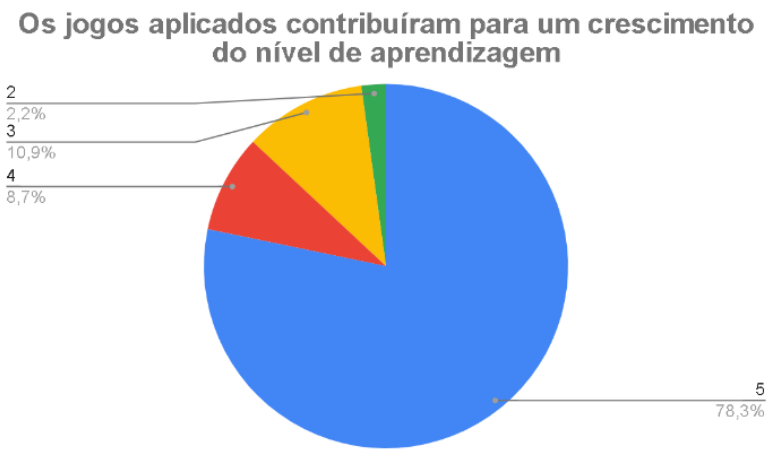

Fonte: Os autores (2021)

Quando questionados acerca da percepção sobre a Aprendizagem Baseada em Jogos, $80 \%$ dos discentes afirmaram que a mesma os incentivou a participar das atividades desenvolvidas (figura 6) Além disso, $80 \%$ concordam totalmente que a aplicação de jogos contribui no armazenamento do conteúdo visto em sala de aula (figura 7). 
Figura 6 - Incentivo da ABJ na participação.

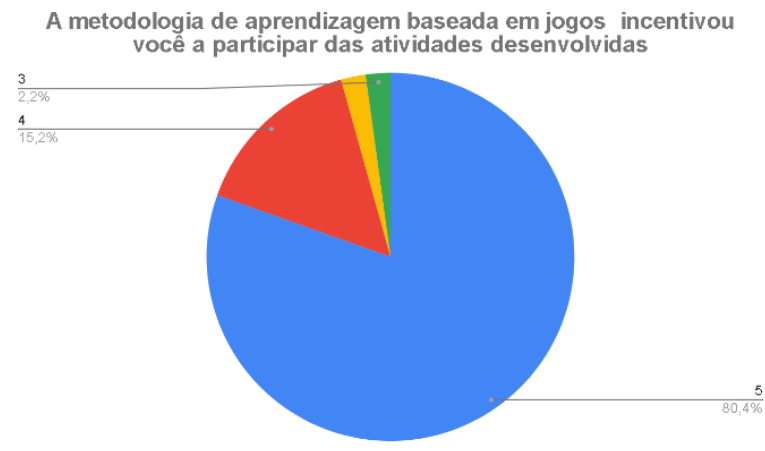

Fonte: Os autores (2021)

Figura 7 - Contribuição dos jogos no aprendizado.

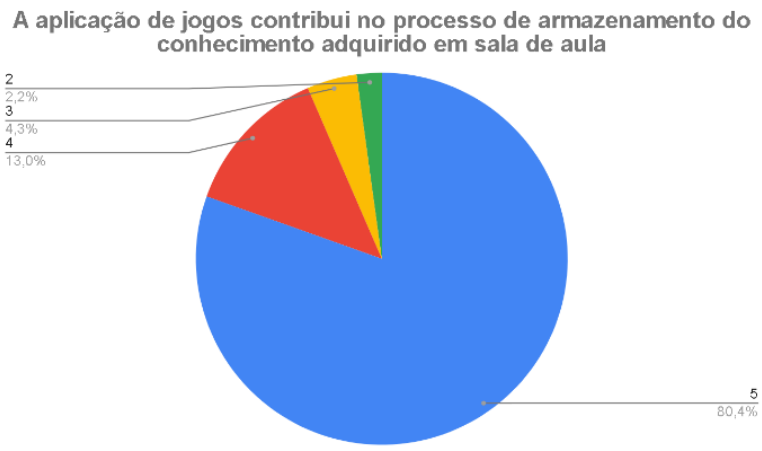

Fonte: Os autores (2021)

Por fim, a maioria dos alunos respondeu positivamente acerca da percepção dos benefícios da Aprendizagem Baseada em Jogos (figura 8). Isso evidencia que os alunos estão abertos a romper com os modelos tradicionais de ensino e conseguem perceber eficácia na $A B J$.

Figura 8 - Incentivo da ABJ na participação

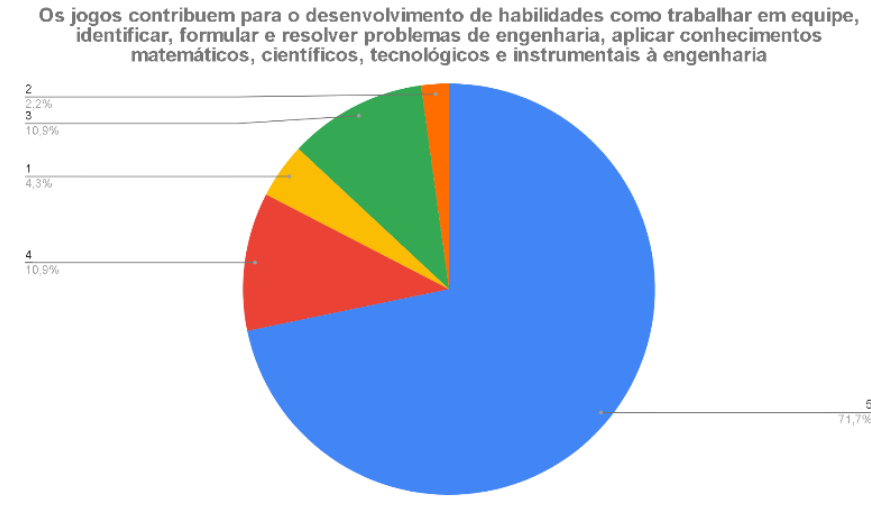

Fonte: Os autores (2021) 


\section{CONSIDERAÇÕES FINAIS}

O presente artigo teve como objetivo compartilhar uma aplicação da Aprendizagem Baseada em Jogos e analisar a percepção dos estudantes acerca da metodologia e sua aplicação no contexto do ensino remoto. Os resultados mostraram que os discentes consideraram os jogos divertidos e que as atividades no Kahoot contribuíram para consolidar os assuntos vistos nas aulas. Os alunos afirmaram também que haver uma premiação relacionada a atividade incentivou a participação destes e mostraram preferência por aplicações assíncronas dos jogos.

Os resultados também reforçam a percepção positiva dos estudantes acerca da metodologia Aprendizagem Baseada em Jogos, sendo essencial que os alunos conheçam e se tornem promotores dessa metodologia, assim como de outras formas de inovações pedagógicas. A pesquisa evidencia que os alunos conseguem reconhecer os benefícios e perceber a importância de romper com modelos tradicionais de ensino.

Por fim, o artigo reforça o uso do Kahoot como ferramenta de suporte ao ABJ no contexto remoto, que é capaz de proporcionar momentos de diversão e fomentar a motivação dos estudantes pelo conteúdo lecionado.

\section{Agradecimentos}

Os autores agradecem à UFC pela concessão de bolsa de monitoria pelo Programa de Iniciação à Docência (PID), ao CNPq pela bolsa de mestrado do penúltimo autor, ao grupo de Logística e Redes de Infraestruturas (LRI) por todo o suporte no desenvolvimento da disciplina e aos alunos, que contribuíram participando da pesquisa.

\section{REFERÊNCIAS}

BARDINI, Vivian Silveira dos Santos; SPALDING, Marianne. Aplicação de metodologias ativas de ensino-aprendizagem: experiência na área de engenharia. Revista de Ensino de Engenharia, v. 36, n. 1, 2017.

CONTRERAS, Ruth Sofhía et al. Aprendizagem baseada em Jogos Digitais Entrevistas com professores que utilizam jogos digitais em suas práticas educativas. 2013.

DA SILVA DAMACENO, Érick William et al. ESTUDO DO USO KIT MOLA ESTRUTURAL NO ENSINO DE ENGENHARIA CIVIL. PI-Pesquisa e Inovação, v. 1, n. 1, p. 38-50, 2019.

DE CARVALHO, Carlos Vaz. Aprendizagem baseada em jogos-Game-based learning. In: II World Congress on Systems Engineering and Information Technology. 2015. p. 176-181.

GUIMARÃES, Gilselene Garcia. A APLICAÇÃO DO MÉTODO DESIGN THINKING NO CURSO DE ENGENHARIA CIVIL: UMA EXPERIÊNCIA NO DESAFIO DE CRIAR E INOVAR O COMPLEXO PROCESSO DE ENSINO E APRENDIZAGEM DA DISCIPLINA DE CÁLCULO. Revista de Ensino de Engenharia, v. 39, 2020.

GUSSO, Hélder Lima et al. Ensino superior em tempos de pandemia: diretrizes à gestão universitária. Educação \& Sociedade, v. 41, 2020. 
PRENSKY, Marc. Fun, play and games: What makes games engaging. Digital gamebased learning, v. 5, n. 1, p. 5-31, 2001.

PAIVA, Carlos A.; TORI, Romero. Jogos Digitais no Ensino: processos cognitivos, benefícios e desafios. XVI Simpósio Brasileiro de Jogos e Entretenimento Digital, p. $1-4,2017$.

\title{
ANALYSIS OF THE APPLICATION OF GAME-BASED LEARNING IN THE REMOTE LEARNING CONTEXT: THE CASE OF THE SUBJECT PROJECT AND CONSTRUCTION OF ROAD INFRASTRUCTURE
}

\begin{abstract}
In the teaching-learning context of engineering, there have been more and more debates that question the traditional and merely expository models of teaching. Debates about the student's role in the classroom and the motivation of the students become even more relevant in the context caused by the pandemic of COVID-19. With the suspension of face-to-face classes, teachers and students were forced to migrate to online platforms, continuing their didactic activities through remote education. At the center of these debates, there is the rise of several teaching methodologies that seek to involve students, placing them as protagonists of learning. In this line, the Game-Based Learning stands out in this work, a methodology referring to the use of the power of motivation and entertainment of games aimed at teaching. Thus, the objective of this article is to share an application of the pedagogical strategy of learning based on games and to evaluate its contribution in a class of the discipline of Design and Construction of Road Infrastructure (PCIV) offered in the totally remote model in 2020. For the application of the games, the kahoot tool for the application of rapid tests of multiple choice about the content seen in the course was used. The results reinforce the students' positive perception about the Game-Based Learning methodology since the students considered the games fun and the activities contributed to consolidate the subjects seen in the classes.
\end{abstract}

Keywords: Remote Teaching. Game-based Learning. Civil Engineering 